\title{
AUFARBEITUNG UND INTERPRETATION VON KONTEXTEN IM ARTEMISION VON EPHESOS Anmerkungen zu Hephaistos 21/22, 2003/04, 169-200
}

In seinem Artikel zur »Kontextualisierung im Artemision von Ephesos« hat sich Michael Weiß1 mit der vierzigjährigen Grabungs- und Forschungstätigkeit von Anton Bammer im Artemision von Ephesos auseinandergesetzt ${ }^{1}$. W(eißl) versucht darin, auf 27 Textseiten und mit 5 schematischen Abbildungen die Kernaussagen eines wissenschaftliches Lebenswerkes »zu evaluieren«, das Bammer auf mehreren hundert Seiten dargelegt hat ${ }^{2}$. W. macht kein Hehl daraus, daß das Ergebnis seiner 'Evaluierung' von Anbeginn feststeht, denn erklärtes Anliegen ist es, die »empirische oder spekulative Argumentationsgrundlage herauszuarbeiten $\aleph^{3}$, auf der das von Bammer entworfene "pluralistische Interpretationsmodell der Artemisiongrabung $\aleph^{4}$ basiert, dem W. ein »Entwicklungsmodell« gegenüberstellt, das im Ansatz auf Vorstufen bei D. G. Hogarth zurückgeht $^{5}$.

Konkrete archäologische Befunde oder Funde aus dem Artemision behandelt W. nur kursorisch. Anders wäre es im Rahmen eines Aufsatzes mit einigen wenigen schematischen Abbildungen auch nicht zu verwirklichen gewesen, denn dazu hätte es einer ausführlichen Dokumentation und Diskussion bedurft. W.s Anliegen ist letztendlich ein grundsätzliches: Im Zentrum seines komplexen Traktats stehen Fragen der Erkenntnistheorie und eine Kritik an organisatorischen Strukturen innerhalb der Archäologie, die »am Beispiel des Artemisions von Ephesos ... demonstriert werden « sollen ${ }^{6}$. W. wendet sich ganz allgemein gegen die »Konventionen der archäologischen Feldforschung ${ }^{7}$, deren Problem er in der hierarchischen Struktur von wissenschaftlichen Unternehmungen sieht, die er im Fachgebiet der Klassischen Archäologie als besonders ausgeprägt empfindet, gerade innerhalb des deutschen Sprachraumes ${ }^{8}$ : »Dem Grabungsleiter wird von den verantwortlichen Institutionen das Publikationsrecht und die damit verbundene Kontextualisierung ad personam übertragen. $\ll^{9} \mathrm{~W}$. versteht die Person des 'Ausgräbers' bzw. 'Grabungsleiters' als Antipoden des wissenschaftlichen Diskurses, da »viele >Ausgräber ‘... kaum einen Nutzen darin sehen dürften, durch offene Diskussionen auf strukturelle oder methodische Schwachpunkte aufmerksam zu machen ${ }^{10}$. Sie würden eine »fundierte Kritik an den Grabungs-

\footnotetext{
1 M. Weiß1, Hephaistos 21/22, 2003/04, 169-200.

2 Ebenda 181. Es ist bemerkenswert, daß W. genau an der Stelle, wo er die Notwendigkeit einer Evaluierung »vor der Publikation >abschließender Grabungsberichte« postuliert und sich selbst zum Evaluator ernennt, auf den »Bericht der internationalen Evaluatorengruppe«, bestehend aus P. Ducrey (Lausanne), R. Fleischer (Mainz), H. P. Isler (Zürich) und C. Nylander (Stockholm/Rom), verweist, die 1993 vom Bundesminister für Wissenschaft und Forschung eingesetzt wurde, um die österreichischen archäologischen Forschungen in Ephesos zu begutachten, s. AnzWien 131, 1994, 309-342.

3 Weiß1 (Anm. 1) 181.

4 Ebenda 172.

5 Ebenda 177 f. Bereits vor jeder Diskussion konstatiert W.: »Der archäologische Befund und die Schriftquellen scheinen für die kontinuierliche Bauentwicklung eines einzigen Kultbaus zu sprechen.«

${ }^{6}$ Ebenda 180.

7 Ebenda 180.

8 Ebenda, Anm. 54.

9 Ebenda.

10 Ebenda.
} 
ergebnissen« dadurch unterbinden, daß sie die dazu notwendige »genaue Kenntnis der archäologischen Evidenz« monopolisierten ${ }^{11}$. W. unterstellt damit, daß ein 'Ausgräber' ein gleichsam natürliches Interesse daran habe, Dokumentationen zurückzuhalten, um seine eigene Interpretation in der »autorisierten Grabungspublikation« unangreifbar zu machen ${ }^{12}$.

Die »Evaluierung von Grabungsergebnissen und ihre Falsifikation«, die W. theoretisch für jedes archäologische Projekt fordert und die seiner Meinung nach »keinesfalls vom Ausgräber selbst vorgenommen werden kann, sondern in kontroversiellem Sinne von kritischen Fachkollegen unternommen werden muß « ${ }^{13}$, führt er im Anschluß für das ephesische Artemision selbst durch. Die »Grundlage« dazu bilden die »neuen Erkenntnisse«, die »die langjährige Fundbearbeitung und die Aufarbeitung der Grabungsdokumentation « erbracht haben ${ }^{14}$. Sie sind für ihn der Anlaß, weshalb er es nicht nur als möglich, sondern sogar als notwendig ansieht ${ }^{15}$, eine Gesamtinterpretation der Grabung »vor der Publikation >abschließender/ Grabungsberichte $\ll^{16}$ zu geben.

Um den »Bewährungsgrad des [von W.] propagierten Modells ... zu bewerten « ${ }^{17}$, ist es notwendig, seine Informationsgrundlage und den Hintergrund seines Entstehens zu kennen. Beides wird von W. nur angedeutet ${ }^{18}$. Als Leiter der Arbeitsgruppe zur Aufarbeitung von Stratigraphie und Keramikfunden der Grabungen im Artemision ${ }^{19}$, deren Mitarbeiter W. von 19982003 war, halte ich es im Sinne einer transparenten Diskussion der $»$ Kontextualisierung im Artemision von Ephesos « für wichtig, an dieser Stelle Informationen nachzuliefern, die zu einer Meinungsbildung über die in Hephaistos 21/22 vorgetragenen Thesen beitragen können.

Die Arbeits- und Publikationsaufgaben der einzelnen Mitarbeiter/-innen innerhalb des Gesamtprojektes »Artemision«, das sich die Auswertung und Veröffentlichung der Ergebnisse der Grabungen des Österreichischen Archäologischen Instituts unter Leitung von A. Bammer zum Ziel gesetzt hat, sind genau definiert. Bei der Planung der Abschlußpublikation der Artemisiongrabungen übernahm W. 1998 zwei Aufgaben: zum einen, in Zusammenarbeit mit dem Verfasser, die Auswertung der Stratigraphie im Bereich des frühen Peripteros und des östlich angrenzenden Geländes, zum anderen einen Beitrag zur »Topographie des Artemisions « ${ }^{20}$, während sich A. Bammer die Vorlage der Architektur vorbehielt. Im Rahmen der Aufarbeitung trug W. den Hauptanteil an der Digitalisierung und Redaktion der umfangreichen Grabungsdokumentation, die heute eine solide Arbeitsgrundlage für die Publikationen der einzelnen Bearbeiter bilden. Als W. am 23. April 2003 auf eigenen Wunsch aus dem Artemisionteam ausschied, um sich, wie er sagte, anderen Forschungsschwerpunkten widmen zu können, wurde eine Reduktion seines Publikationsanteils auf den »Bereich zwischen Peripteros und östlicher Sekosmauer des Kroisostempels" vereinbart, um ihm trotz des vorzeitigen Ausstiegs einen Anteil an der Abschlußpublikation zu ermöglichen ${ }^{21}$. In seinem Beitrag in Hephaistos 21/22 tritt W. allerdings mit dem Anspruch auf, die Stratigraphie der gesamten Grabung und darüber hinaus die Zusammenhänge, die Abfolge sowie die absoluten Datierungen der architektonischen Überreste geklärt zu haben, und das anscheinend im Alleingang, denn die Anteile der übrigen Projektmitarbeiter und -mitarbeiterinnen werden verschwiegen.

11 Ebenda 180. 186.

12 Ebenda 180.

13 Ebenda 186.

14 Ebenda $169 \mathrm{f}$.

15 W. verwendet ebenda 170 die Formulierung: »müssen ... thematisiert und ... diskutiert werden.«

16 Ebenda 181.

17 Ebenda 198.

18 Hinweise sehr allgemeiner Natur finden sich in den ersten beiden Anmerkungen ebenda $169 \mathrm{f}$.

19 Zu Aufgaben und Struktur des Projektes s. ÖJh 71, 2002, 374.

20 Vgl. Projekt Nr. 7743 des Jubiläumsfonds der Oesterreichischen Nationalbank; Aktenarchiv des ÖAI Zl. 722/01: BR. Es handelt sich um die Sondagen 581, 584, 740, 810, 970, 971, 1020, 1021, 1022, 1023, 1030-1039.

${ }^{21}$ Vgl. Aktenarchiv des ÖAI Zl. 480a/03:B-R9. Es handelt sich um die Sondagen 970, 971, 1020, 1021, 1022 und 1023. 
W. veröffentlicht ein Diagramm, das »die kontinuierliche Bauentwicklung« der ergrabenen Architekturen im Artemision nicht nur in eine relativchronologische Abfolge bringt, sondern auch mit absoluten Datierungen versieht ${ }^{22}$. Da darin auch der frühe Peripteros und seine Umbauten auf das Jahrzehnt genau datiert werden, beansprucht diese Tabelle höchste Relevanz für die Frage nach der Entwicklung der frühgriechischen Sakralarchitektur ${ }^{23}$. W. erstellte die von ihm vorgeschlagene relative und absolute Chronologie der Architekturen »aufgrund des stratigraphischen Befundes $\ll^{24}$. Genau betrachtet stützt sich W. allerdings nur auf einen Teil dieses Befundes, nämlich auf die Beschreibung der Schichtenfolge, deren zeichnerische, photographische und deskriptive Dokumentation er für den zentralen und östlichen Grabungsbereich während seiner Projektmitarbeit auswertete. Da W. meinte, eine Gesamtinterpretation ohne Zusammenarbeit mit den Bearbeiterinnen und Bearbeitern der einzelnen Fundgattungen unternehmen zu können, fehlen ihm all jene Informationen des »stratigraphischen Befundes «, die aus den in den einzelnen Schichten enthaltenen Funden stammen, wobei der Keramik aufgrund ihrer Häufigkeit und relativ genauen Datierbarkeit im Artemision die größte Bedeutung zukommt. Durch systematische Beobachtungen zu Fundfrequenz, Erhaltungszustand und Anpassungen von Fragmenten lassen sich Aussagen zu Zusammengehörigkeit, Funktion und Deponierungsgeschichte einzelner Straten gewinnen. Dazu wurde vom Verfasser eine an die spezifische Grabungs- und Dokumentierungssituation am Artemision angepaßte Auswertungsmethode entwickelt ${ }^{25}$. W. jedoch glaubt, auf den keramologischen Teil des stratigraphischen Befundes verzichten zu können und mißachtet damit eine von ihm selbst aufgestellte Forderung: »Eine zeitgemäße Praxis archäologischer Feldforschung muß danach trachten, die Beobachtungen und Interpretationen aller entsprechend ausgebildeten Grabungsteilnehmer und Fundbearbeiter in Einklang zu bringen. ${ }^{26}$ Genau das verabsäumt W. aber und schmälert so entscheidend die Basis, auf die sich seine Interpretationen stützen können.

Keramische Datierungen sind weder monokausal noch punktgenau, sondern hängen von mehreren Faktoren ab wie typologischer und stilistischer Entwicklung, Laufzeiten einzelner Formen und Dekormotive, Benutzungsdauer von Gefäßen, Datierbarkeit von Vergleichsfunden, Erhaltungszustand, Anzahl und Charakterisierbarkeit der in einer Assemblage vertretenen Gefäße etc. Sie bedürfen daher ihrerseits einer eingehenden Diskussion, was nur im Rahmen einer ausführlichen Materialvorlage möglich ist. Auf eine solche aber glaubte W. verzichten zu können $^{27}$ und fühlt sich dennoch in der Lage, in seinem Gesamtentwurf der »Bauentwicklung « auf das Jahrzehnt genaue Datierungen für einzelne Architekturen anzugeben ${ }^{28}$. W. legt dabei nicht offen, in welchen Fällen er sich auf die Auswertung der gesamten Funde einer Sondage stützt und wo seine Datierungsansätze lediglich auf Einzelfunden basieren. Manche von W.s chronologischen Angaben sind überhaupt nicht durch Funddatierungen abgesichert, sondern allein aufgrund der von ihm angenommenen Phasengliederung interpoliert.

22 Weiß1 (Anm. 1) 197 Abb. 5.

23 Zur Stellung des ephesischen Peripteros innerhalb der frühgriechischen Ringhallentempel u. a.: A. Bammer, AnatSt 40, 1990, 137-160 Taf. 11-22 bes. 153. 156; G. Gruben, Griechische Tempel und Heiligtümer ${ }^{5}$ (2001) 381-390; M. Weiß1, ÖJh 71, 2002, 321-327.

24 Weiß1 (Anm. 1) 194; vgl. ebenda 191.

25 M. Kerschner - S. Ladstätter - G. A. Plattner, ÖJh 68, 1999, Beibl. Jahresbericht 1998, 49-54. W. übergeht nonchalant, daß dieser Teil der stratigraphischen Bearbeitung nicht von ihm stammt, ebenso wie er grundlegende Beobachtungen von Kollegen, die bereits vor ihm oder gleichzeitig an der Auswertung der Schichtenfolge arbeiteten, ohne Erwähnung in seiner Datierungstabelle benutzt, vgl. M. Kerschner, ÖJh 66, 1997, Beibl. 85-226; ders. in: B. Rückert - F. Kolb (Hrsg.), Probleme der Keramikchronologie des südlichen und westlichen Kleinasiens in geometrischer und archaischer Zeit. Internationales Kolloquium Tübingen 24. 3.-26. 3. 1998, Antiquitas 3, 44 (2003) 44-50 Abb. 1-5 sowie die Ergebnisse von A. Sokolicek zur Ostmauer des Tempels C - vgl. U. Muss, ÖJh 68, 1999, Beibl. Jahresbericht 1998, 16 - oder von St. Karl zu den östlich an den Peripteros anschließenden Sondagen.

26 Weiß1 (Anm. 1) 186.

27 Ebenda 181.

28 Ebenda 197 Abb. 5. 
Die Grabungen von A. Bammer im Artemision dauerten von 1965-1994 und erbrachten 12152 grabungstechnische Einheiten ('Fundkisten'29), mit deren systematischer Aufarbeitung ab 1995 begonnen wurde. Bisher wurden 5205 charakteristische Einzelgefäße und -fragmente katalogmäßig erfaßt. Da das Ziel des Artemisionprojektes eine kontextuelle Fund- und Befundvorlage ist, wurden jeweils alle charakteristischen Keramikfunde aus den ungestörten Straten einer Sondage aufgenommen. Dabei wurde von der östlichen Grabungsgrenze her begonnen, weil dort die Schichtenfolge am vollständigsten erhalten und am besten dokumentiert ist. Im westlichen Grabungsbereich konnte sich W. nur auf einzelne korinthische Gefäße stützen, die von A. Gasser in einer Gattungsmonographie vorgelegt worden waren ${ }^{30}$. Diese machen aber selbst zur Blütezeit des Imports während der frühkorinthischen Stilphase nicht mehr als 10\% der gesamten Keramikfunde aus den Kontexten im Artemision aus ${ }^{31}$. Damit aber vernachlässigt W. bei seinen Datierungen 90\% der Gesamtfunde, was einen großen Unsicherheitsfaktor darstellt, den A. Gasser im Unterschied zu W. deutlich formulierte ${ }^{32}$.

Wenig fundiert ist auch der Datierungsvorschlag, den W. für den Apsidenbau vor der Südwestecke des 'Kroisostempels' liefert ${ }^{33}$. Er stützt sich allein auf einen Eintrag der Grabungsmitarbeiter J. Fürnholzer und M. Philipp: »An Keramik kam ephesische Ware und Vogelschalen zu Tage.« Es bleibt völlig ungeklärt, in welchem stratigraphischen Verhältnis diese Fragmente zu den architektonischen Resten stehen. Beide keramischen Gattungen haben eine lange Laufzeit: die Vogelschalen reichen von ca. 670-590 v. Chr. (wobei darüber hinaus damit zu rechnen ist, daß kleine Fragmente bei einem ersten Augenschein leicht mit solchen von Vogelkotylen verwechselt werden können, was eine Ausdehnung der Laufzeit nach oben hin bis ca. $750 \mathrm{v}$. Chr. bedeuten würde), die Ephesische Ware (von der nach G. Gürtekin-Demir eine »Ephesianising ware « zu scheiden ist) läßt sich in der zweiten Hälfte des 7. und in der ersten Hälfte des 6. Jahrhunderts v. Chr. nachweisen ${ }^{34}$. Um zu einer verläßlichen Datierung des Apsidenbaus zu gelangen, wird also kein Weg daran vorbei führen, die Keramik und ihre Fundlage im Detail zu bearbeiten. In seinen Datierungen zeigt W. genau jene »selektive Informationsauswahl«, die er an den Vorberichten von A. Bammer kritisiert ${ }^{35}$. W. verkürzt Sachverhalte, die einer ausführlichen Erörterung bedürften, um sein Interpretationsmodell zu unterstützen. Sein Artikel erweist sich damit »als zu reduktionistisch, um die grundlegenden Probleme einer Grabung behandeln zu können $\aleph^{36}$.

W.s Ziel war es, »vor der Publikation >abschließender< Grabungsberichte ..., die Baugeschichte des Artemisions ... zu evaluieren ${ }^{37}$. Unter einer Evaluierung versteht W. nicht nur

29 Zu Begriff und Auswertbarkeit der 'Fundkiste' vgl. Kerschner - Ladstätter - Plattner (Anm. 25) 49-51.

30 A. Gasser, Die korinthische und attische Importkeramik vom Artemision in Ephesos, FiE XII 1 (1989); vgl. Weiß1 (Anm. 1) 189. 191. Ein logischer Fehler liegt ebenda 191 vor, wenn W. meint: »Das Fundament des Hekatompedos liegt in einer Aufschüttung, deren Entstehung aufgrund der frühkorinthischen Keramikfunde nach 590 v. Chr zu datieren ist.« Als terminus post quem kann hier jedoch nicht das Ende der frühkorinthischen Stilphase (um 595/90 v. Chr.) gelten, sondern deren Beginn (um 620/15 v. Chr; beide Zeitansätze nach D. A. Amyx, Corinthian Vase-Painting of the Archaic Period [1988] 428), da ja die frühkorinthischen Gefäße sehr kurz nach ihrer Entstehung unter die Erde gelangt sein können.

31 Vgl. Kerschner (Anm. 25:1997) Beibl. 205. 208; zur Entwicklung des korinthischen Keramikimports nach Ephesos: Gasser (Anm. 30) 70.

32 Gasser (Anm. 30) 71: „Soweit es mir nach den nur Teilgebiete des gesamten Fundmaterials umfassenden Studien möglich ist, kann ich jüngeres, später als vielleicht ins erste Jahrzehnt des 6. Jhs. v. Chr. datierbares Material für die Schichten unter dem unteren >gelben Boden` ausschließen.«

33 Weiß1 (Anm. 1) 175 mit Anm. 26.

34 Zur Datierung der Vogelschalen: J. N. Coldstream, Greek Geometric Pottery (1968) 298-301; Kerschner (Anm. 25: 1997) 189 f.; zur Datierung der Ephesischen Ware: C. H. Greenewalt, Jr., California Studies in Classical Antiquity 6, 1973, 116 f.; R. G. Gürtekin-Demir, AnatSt 52, 2002, 114; zur 'Ephesianising ware': ebenda 115-119.

35 Weiß1 (Anm. 1) 186.

36 Weiß1 (Anm. 1) 200.

37 Ebenda 181. Ähnlich formuliert W. dieses Ziel in dem Antrag zu seinem zur Zeit an der Universtität Tübingen laufenden Projekt: »Zukünftige Grabungspublikationen werden auf der entwicklungsgeschichtlichen Analyse des Artemisions aufbauen können.«, s. http://www.fwf.ac.at/de/abstracts s. v. Erwin-Schrödinger-Stipendien J2376. 
Kritik an bereits geäußerten Hypothesen, sondern die Präsentation eines fertigen Gegenmodells. Wie ich mich hier zu zeigen bemühte, ist es beim jetzigen Stand der Auswertung und in der vorgebrachten reduktionistischen Form gar nicht möglich, ein Erklärungsmodell mit allumfassendem Gültigkeitsanspruch zu entwickeln. Ich stimme W. zu, daß »erst die langwierige Analyse der archäologischen Evidenz ... nachvollziehbare Aussagen« erlauben wird ${ }^{38}$. Nur eine umfassende Vorlage der relevanten Funde und Befunde kann die notwendige Basis schaffen für eine Diskussion der Entwicklung des Heiligtums. Michael Weiß1 hat eine Lösung durch ein Hysteron-Proteron angestrebt, dem die Beweiskraft fehlt.

Dr. Michael Kerschner

Österreichisches Archäologisches Institut, Franz Klein-Gasse 1, A-1190 Wien

E-Mail: michael.kerschner@oeai.at

38 Weiß1 (Anm. 1) 200. 
